\title{
A Survey on Optical Fiber Sensors for Telemedicine Applications
}

\author{
Hadeel Kassim AL-Jobouri \\ Medical Engineering Department/College of Engineering / Al-Nahrain University-Baghdad \\ Ph.D. Student at Yildrim Beyazit University/Institute of Science \& Technology -Ankara \\ *Corresponding author: hadeel_bme77@yahoo.com
}

Copyright (C) 2013 Horizon Research Publishing All rights reserved.

\begin{abstract}
In this paper most of the important telemedicine applications with optical fiber sensor were introduced, and they included two techniques of using optical fiber sensors with monitoring and treating patients remotely through the operation. Optical sensors with MRI compatibility have been used in these two fields through catheter and biopsy techniques. This survey is the first one in which the optical fiber sensors in the field of biomedical engineering applications has been used.
\end{abstract}

Keywords Fiber-Optic Sensor, Catheterization, Optical Sensors with MRI Compatibility, Needle Biopsy

\section{Introduction}

As was mentioned above two fields of optical fiber sensors with biomedical technology application have been introduced, which are optical fiber sensors with MRI compatibility and an electronic nose.There are many applications (diagnosis or treatment) in using optical fiber sensors with MRI compatibility, and in this paper the most important two of them will be introduced: catheter and biopsy.

\subsection{Catheterisation}

Cardiac catheterization (heart cath)is the insertion and progression of a catheter into the body which achieved from a small incision on the groin Fig.1 (upper thigh), the arm, or the neck of the patient [1]. This is done for both diagnostic and interventional purposes and is considered to be a minimally invasive technique that is usually carried out under image guidance of X-ray fluoroscopy.Unfortunately, $\mathrm{X}$-ray guided cardiac catheterisation has poor soft-tissue contrast which makes the positioning of guide wires, catheters, balloons, and interventional devices difficult. Interventional MRI is safe and practical in a clinical setting allowing better three dimensional visualization of the anatomy with superior soft tissue contrast, while avoiding ionizing radiation.

The Magnetic Resonance Imaging (MRI) compatible fibre-optic sensor for integration with catheters allowing the detection of contact forces between blood vessel walls and the catheter tip [2].

These forces are a combination of two secondary forces. The one originates from the friction of the catheter with the blood vessel walls and the other from the contact of the catheter-tip with the blood vessels.

The Magnetic Resonance Imaging (MRI) compatible fibre-optic sensor for integration with catheters allowing the detection of contact forces between blood vessel walls and the catheter tip [3].

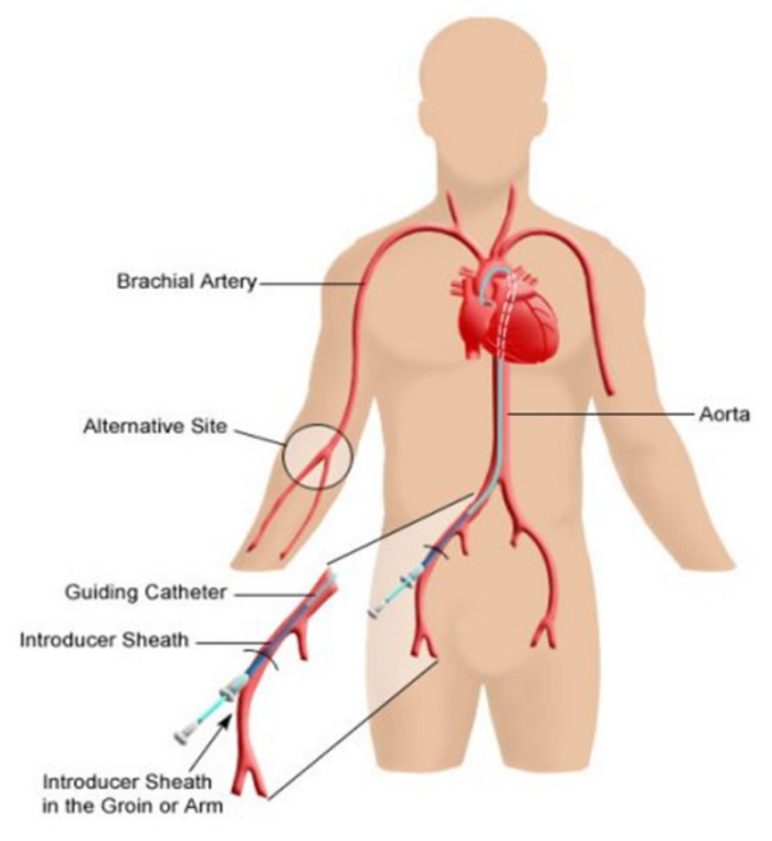

Figure 1. Catheter Insertion

\subsection{Biopsy:}

Biopsy is a procedure that uses very thin needles or guide wires to mark the location of an abnormal area of tissue so it can be surgically sampled Fig.2 [4, 5]. 


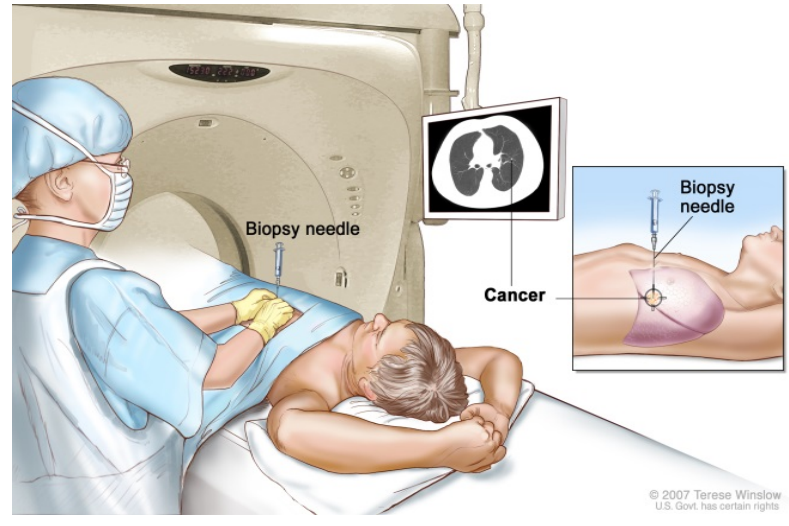

Figure 2. Surgical Biopsy Operation

Radiofrequency ablation (RFA) has in recent years become a popular treatment for primary tumors in the breast, kidney, liver, and etc.However, traditional approaches of guidance such as ultrasound and computed tomography (CT) fail to provide satisfactory placement precision. Although MRI guidance offers the ability to evaluate the completeness of the RFA, those procedures that are currently done using MRI are performed under "image guidance" rather than "continuous imaging". For this reason optical fiber sensors used with MRI compatibility, also the materials used here must be a low magnetic susceptibility making this sensor fully MRI-compatible and inexpensive Fig.3.

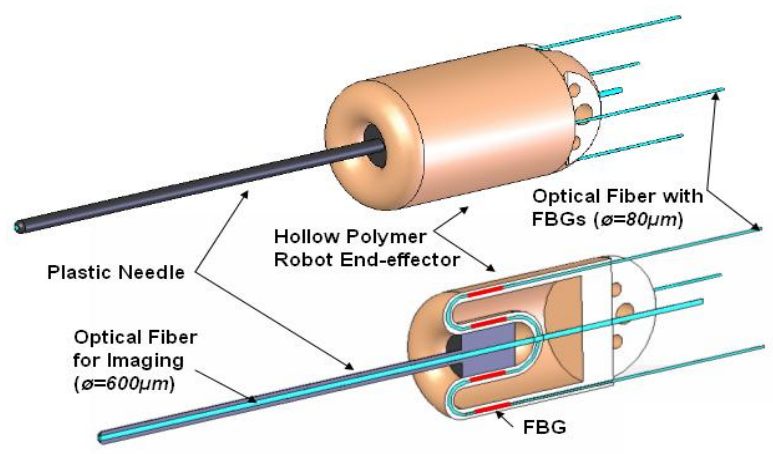

Figure 3. Optical fiber sensors used with MRI compatibility

\section{Sensor with MRI Compatibility Structure}

In this paper the feasibility study of a three axes fibre-optic tip catheter force sensor and 3-DOF fibre-optic force sensor are presented.

Catheterisation is a minimally invasive approach which targets to offer treatment or examination to tissues and organs using existing routes inside a human body. Routes such as the blood vessels are used to allow thin, long and flexible catheters to reach targeted settings within the body and their diameter does not exceeding 3-4 $\mathrm{mm}$ [1]. In a catheterisation the restrictions for the size of instruments to be used originates in the size of the blood vessel. Therefore, as cameras are almost impossible to be inserted, other visualisation methods are used. Fluoroscopy and Computed Tomography (CT) are the two most common methods used today by cardiologists. These techniques employ ionizing technology, such as X-rays, to represent the inner human structure. However, their use is hazardous for the human body. In addition, the quality of the tissues and organs in the returned images is poor. In order to enhance them the catheter has to inject a contrast medium inside the area of interest.

Lately, scientists are interested in substituting these methods with others which have distinct advantages; one of these methods is the MRI. MRI is making use of magnetic fields to produce the images. As a result, these images are giving three dimensional representation of the internal body structure with superior quality and without emitting hazardous ionising radiations.

Despite the advantages of an MRI scanner, the use of tools and instruments inside its area of operation which contain materials that could affect the homogeneity of the magnetic field must be avoided. Furthermore, the insertion of conductive wires can potentially work as an MR-antenna, which can result in potential heating effects at the tip of the wire during MR-scanning. Hence, metal materials are ineffective, as either distort the images, producing artefacts, or heating up effects putting in health risks the patients [6]-[7].

\subsection{A Three Axes Fibre-Optic Tip Catheter Force Sensor Structure:}

Three plastic optical-fibres cables are aligned inside a plastic catheter in a circular pattern. A reflective circular mirror with a hole in the middle is attached to a separate small part of the catheter tip, which is connected with a small deformable material to the aligned optical-fibres Fig.4.

In this manner a force at the catheter tip leads to a deformation of the elastic material and thus a modulation of the light yields, this is sent and received through the optical-fibres.

An electronic circuit amplifies the retrieved light signal and the output voltage is used to classify the forces on the tip. The materials used are of the shelf and have a low magnetic susceptibility making this sensor fully MRI-compatible and inexpensive.

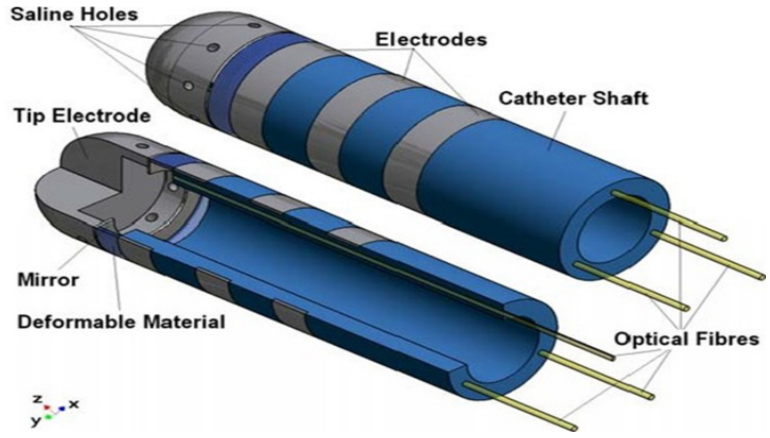

Figure 4. Drawing views of a catheter illustrating the proposed concept of the three axes fibre-optic force sensor. 
The sensor integrated with the catheter is shown in Fig.5(top). The plastic optical-fibres are aligned inside the two lumens in such way to create a circular pattern with 120 degrees spacing between them.

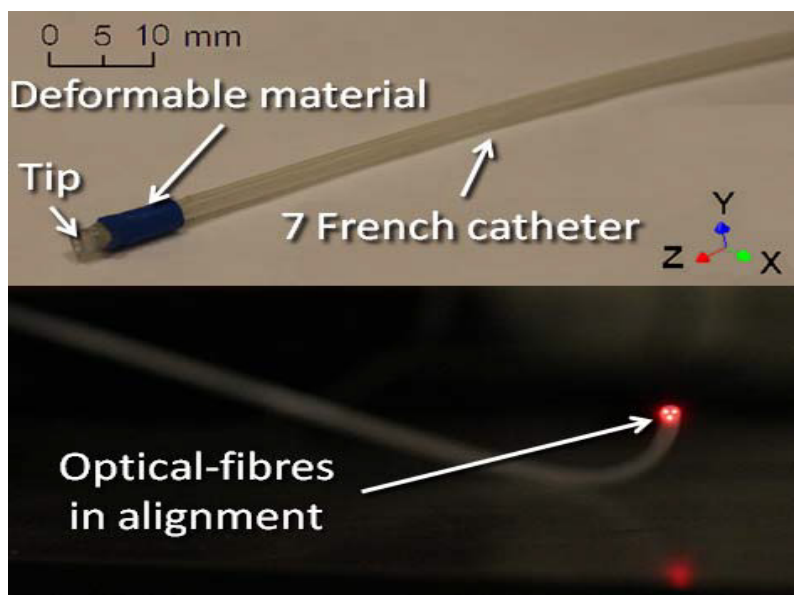

Figure 5. Top: The fibre-optic sensor inside a catheter, without Electrophysiology ablation (EP) electrodes. Bottom: The alignment of the optical-fibres inside the same catheter.

The optical-fibres illuminating inside the catheter can be seen in Fig.5 (bottom), whereas in Fig.6 (left) the alignment within the lumens of the catheter is shown.

In addition, a short in length part of the plastic catheter is cut prior to the optical-fibres alignment, in order to be used as the tip of the catheter-sensor. The circular mirror is positioned concentrically at one of the two cylindrical flat surfaces of the short catheter part, leaving the way free to the lumens.

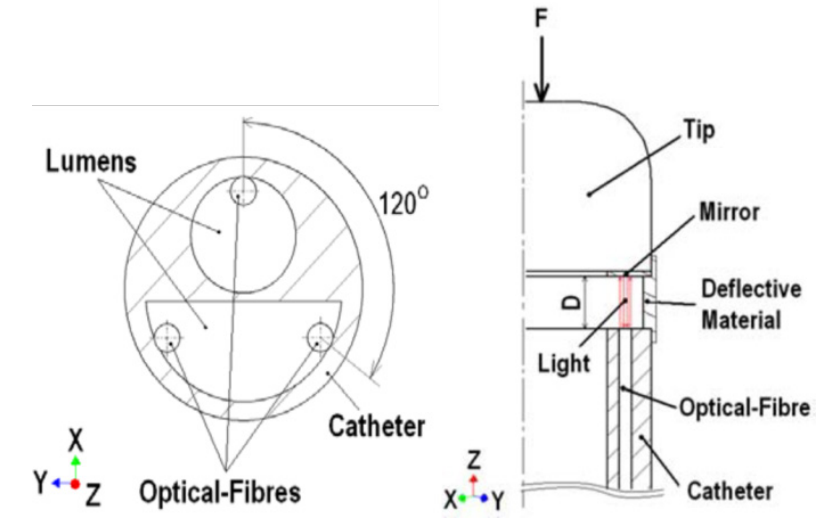

Figure 6. Left: A horizontal section view of the catheter showingthe shape of the two lumens and the position of the optical-fibres.Right: A vertical partial section view of the fibre-optic catheter sensorshowing one of the three optical-fibres emitting and receiving light ata distance $\mathrm{D}$ from the mirror.

The deformable material is obtained in a form of a thin film and is wrapped and glued concentrically around the main part of the catheter and the short catheter part, which accommodates the mirror. After the wrapping the catheter's tip diameter increased. With this technique the two parts are connected leaving $1 \mathrm{~mm}$ of space between the aligned optical-fibres and the reflector. The distance denoted with the letter ' $D$ ' in Fig.6 (right) shows the free space previously described.

\section{$>$ Principle of operation:}

The principle of operation for this fibre-optic scheme is based on light intensity modulation caused by the change in distance of the reflector when a force is applied at the tip of the catheter. Each one of the optical-fibres is interrogated by a red LED.

Fibre-optic couplers are employed to emit and receive the reflected light through the same single integrated optical-fibre within the catheter.

The couplers are used to:

- Ensure less occupied space by optical-fibres within the catheter.

- Increase the amount of received/reflected light signal.

At the end of the optical couplers a dedicated amplifier electronic circuit is responsible to collect the light signal using photodiode detectors. The light signal is converted in a voltage signal and passes through stages of amplification.

The three optical-fibres are positioned in such way to return, after calibration, the vector of the force in a Cartesian coordinate system with $\mathrm{X}, \mathrm{Y}$ and $\mathrm{Z}$ components. The $\mathrm{Z}$ component is calculated from the average change in distance ' $\mathrm{D}$ ' of the reflected light from all the optical-fibres when a force is applied in the tip vertically. The remaining $\mathrm{X}$ and $\mathrm{Y}$ components occur when forces are applied at an angle from the vertical $\mathrm{Z}$ axis at the tip.

\section{Test Bench:}

To investigate the force output of the sensor in axial loading, a test bench was employed.The suggested test bench consisted of a vice, which act as the rigid support for the catheter, an axes force/torque sensor, a robotic manipulator, the optical-fibre amplifying circuit and a data acquisition.

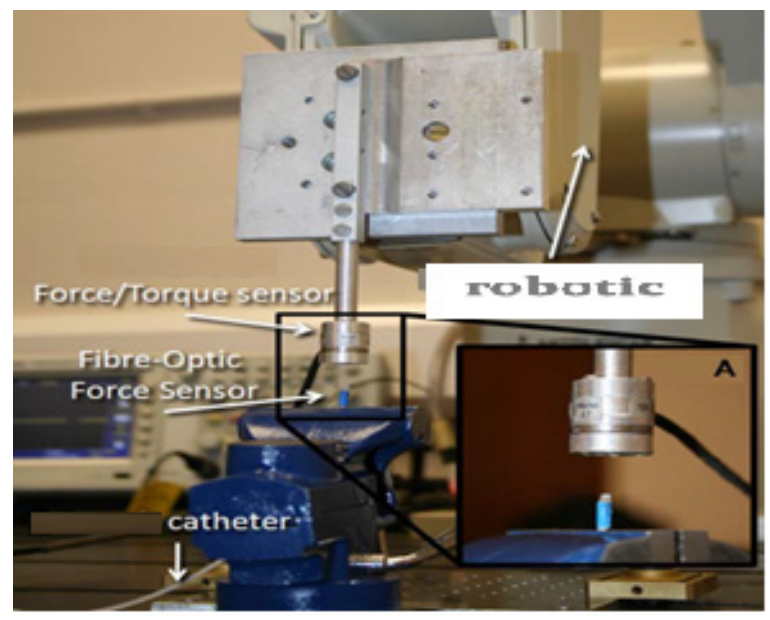

Figure 7. Correlating the forces applied to the fibre-optic sensor with the voltage output of the optical-fibres. The test bench and a close-up view (A) of the nano17 and the produced sensor.

The sensor was placed in the support vertically, having the 
tip looking upwards and leaving some $\mathrm{mm}$ to stand out as shown in Fig.7. The force sensor was mounted on the manipulator aligned above the developed sensor. Using this configuration the manipulator could translate with a constant low speed in the axial direction and towards the fibre-optic sensor deforming the elastic connecting material, closing slowly the gap between the reflector and the light emitting optical fibres.

The light intensity variation, after the signal amplification, was constantly recorded using the data acquisition card. In addition, the force measurements obtained by the sensor, while exerting force to the developed sensor, were also recorded.

\subsection{3-DOF Fibre-Optic Force Sensor Structure:}

Ateleoperated multi-DOF

device

for biopsy/Radiofrequency ablation (RFA) is MRI compatible. Whereby identification of tumor boundaries, placement of the needle, assessment of placement accuracy, ablation, and assessment of ablation accuracy can be done in one sitting, without removing the patient from the scanner or disrupting tumor location, as shown in Fig.8.

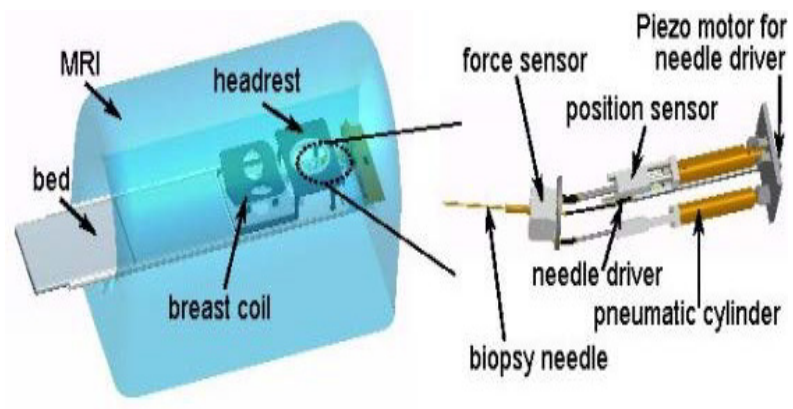

Figure 8. The robot inside the MRI bore[7]

To actuate the device, we chose pneumatic cylinders and piezo motor to ensure MRI-compatibility and maintain MR image quality. We must build with non-ferromagnetic material biopsy robot, as shown in Fig.9.

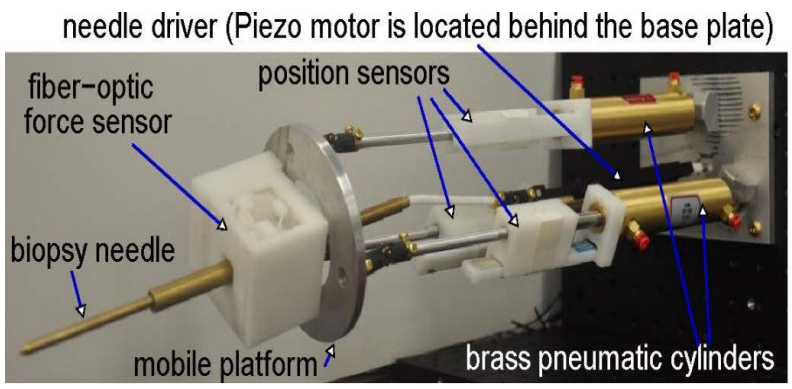

Figure 9. The 4-DOF MRI-compatible breast biopsy robot [8]

It consists of a parallel mechanism that is actuated with three pneumatic cylinders and a needle driver that is actuated with a piezo motor. This device is capable of adjusting needle orientation and performing the biopsy needle insertionas shown in Fig.10.

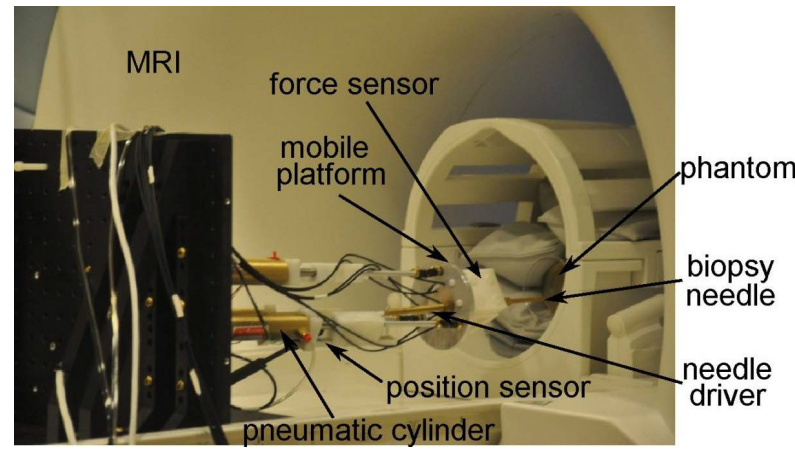

Figure 10. Experimental setup in the MRI [8]

A 3-DOF fibre-optic force sensor with optical approach is proposed because it is inherently MRI-compatible as signals are transmitted in the form of light which eliminates the existence of electrical wires inside the MRI room as illustrated in Fig. 11.

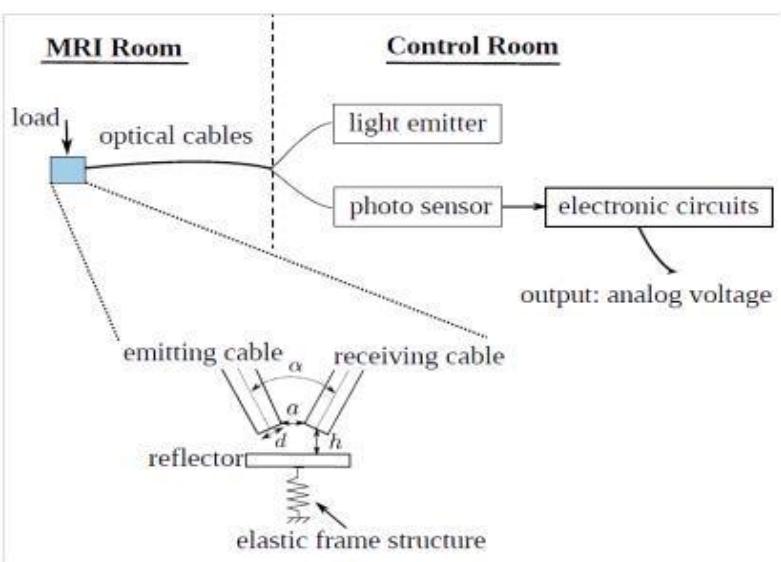

Figure 11. Overview of MRI Compatible Fiber-optic Force Sensor Using Reflective Intensity Measurement [9]

The same process and as I mentioned previously with fibre-optic tip catheter force sensor, also here a force acting on the force sensor causes a deformation in the elastic frame structure, resulting in a displacement in the reflector.

A topology optimization technique is also used to provide a systematic algorithm aiding engineers in designing the elastic frame structures that are needed.

Fig. 12 shows the first and final photo of the prototype and Fig.13 illustrates the MR image quality of the force sensor.
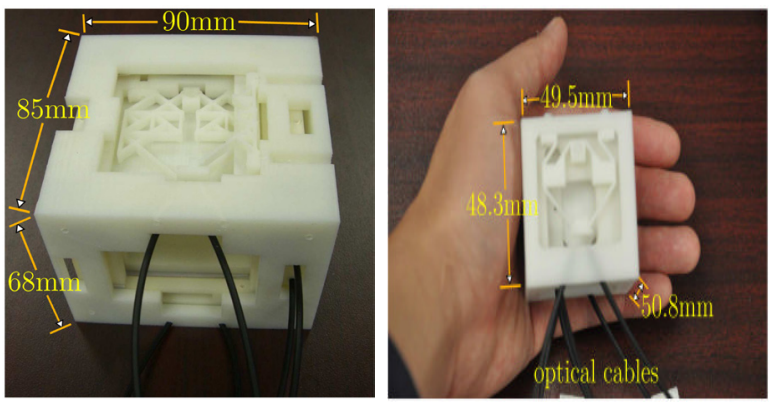

Figure 12. Left: The first; Right: The final Prototype of the 3-DOF MRI Compatible Fiber-optic Force Sensor [9] 


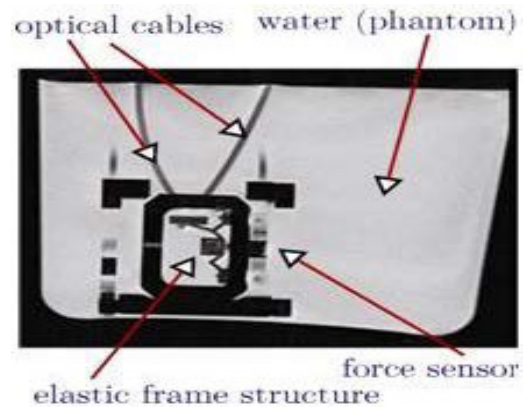

Figure 13. Magnetic resonance Image of the Fiber-optic Force Sensor[9]

\section{Conclusion}

This is the first paper was introduced the most important telemedicine applications with optical fiber sensor. In this paper two fields on optical fiber sensors with biomedical technology applications and continuity with patients remotely were introduced. These two fields have used optical sensors with MRI compatibility and they are catheter and biopsy.

Really, there are several systems have been developed to enhance catherisations, nevertheless, most of the solutions given today by scientist do not take into account the ease in manufacturing, the costs for production and the MRI-compatibility.

At the end of the optical couplers in a fibre-optic tip catheter, a dedicated amplifier electronic circuit is responsible to collect the light signal using photodiode detectors. The light signal is converted in a voltage signal and passes through stages of amplification to ensure reduction of the electrical.

In fibre-optic force sensor, there is a tele-operation system with haptic interface, this device in the MRI bore is a slave device and will be teleoperated by a master device placed in the MRI control room.

I believe that the integration of haptic interface will improve the success rate of the procedures to some extent by helping to characterize the tissue type (normal, fat, cancerous, etc.) during biopsy/RFA needle insertion tasks. Such master device is currently under development.

\section{REFRENCES}

[1] F. WeiXing, W. HuanRan, G. ShuXiang, W. KeJun, Y. XiuFen,"Realization of a Catheter Driving Mechanism with Micro tactilesensor for Intravascular Neurosurgery",IEEE InternationalConference on Robotics and Biomimetics, pp. 1628-1633, (2006).

[2] R. Razavi, D.L. Hill, S.F. Keevil, M.E. Miquel, V. Muthurangu, S.Hegde, K. Rhode, M. Barnett, J. van Vaals, D.J. Hawkes, E. Baker, "Cardiac catheterisation guided by MRI in children and adults withcongenital heart disease", Lancet, Vol. 362, no. 9399, pp.1877-82, (2003).

[3] S. Weiss, T. Kuehne, F. Brinkert, G. Krombach, M. Katoh, T.Schaeffter, R.W. Guenther, A. Buecker," In vivo safe catheter visualizationand slice tracking using an optically detunable resonant marker", MagnReson Med. Vol. 52, no.4, pp.860-8, (2004).

[4] G. S. Fischer, I. I. Iordachita, C. Csoma, J. Tokuda, S. P. DiMaio,C. M. Tempany, N. Hata, and G. Fichtinger, "Mri-compatible pneumaticrobot for transperineal prostate needle placement", IEEE/ASME Transactions on Mechatronics, vol. 13, (2008).

[5] www.vascularinterventions.net

[6] S. Weiss, P. Vernickel, T. Schaeffter, V. Schulz, B. Gleich."Transmission line for improved RF safety of interventional devices", Magnetic Resonance in Medicine, Vol. 54, no.1, pp. 182-189, (2005).

[7] N. Yu, R. Riener. "Review on MR-Compatible Robotic Systems",TheFirst IEEE/RAS-EMBS International Conference on BiomedicalRobotics and Biomechatronics, pp. 661-665, Pisa, Italy 20-22 Feb. (2006).

[8] Bo Yang, U-Xuan Tan, Alan McMillan, RaoGullapalli, and Jaydev P. Desai, "Design and implementation of a pneumatically-actuated robot for breast biopsy under continuous MRI", In IEEE International Conference on Robotics and Automation, pp. 674-679, Shanghai, China, (2011).

[9] U-Xuan Tan, Bo Yang, RaoGullapalli, and Jaydev P. Desai, "Tri-Axial MRI Compatible Fiber-optic Force Sensor", IEEE Transactions on Robotics, vol. 27, no. 1, pp. 65-74, (2011). 